REVIEW

\section{Pain regulation by non-neuronal cells and inflammation}

\author{
Ru-Rong Ji, ${ }^{1,2 *}$ Alexander Chamessian, ${ }^{1}$ Yu-Qiu Zhang ${ }^{3}$
}

Acute pain is protective and a cardinal feature of inflammation. Chronic pain after arthritis, nerve injury, cancer, and chemotherapy is associated with chronic neuroinflammation, a local inflammation in the peripheral or central nervous system. Accumulating evidence suggests that non-neuronal cells such as immune cells, glial cells, keratinocytes, cancer cells, and stem cells play active roles in the pathogenesis and resolution of pain. We review how non-neuronal cells interact with nociceptive neurons by secreting neuroactive signaling molecules that modulate pain. Recent studies also suggest that bacterial infections regulate pain through direct actions on sensory neurons, and specific receptors are present in nociceptors to detect danger signals from infections. We also discuss new therapeutic strategies to control neuroinflammation for the prevention and treatment of chronic pain.

〔 linically, inflammation is characterized by five cardinal signs: rubor (redness), calor (increased heat), tumor (swelling), dolor (pain), and functio laesa (loss of function). Acute inflammation is a protective response involving immune cells, blood vessels, and molecular mediators (inflammatory mediators). The function of inflammation is to eliminate the initial cause of cell injury and initiate tissue repair. Acute pain, also known as nociceptive pain, is a cardinal feature of inflammation. The majority of known inflammatory mediators cause pain by binding to their receptors on nociceptive primary sensory neurons in the peripheral nervous system (PNS) (nociceptors) that innervate injured skin, muscle, and joint tissues (Fig. 1) (1-3). Once thought to be a passive process, the resolution of acute inflammation is now recognized as a distinct, active process involving specialized pro-resolution mediators (SPMs) such as resolvins, protectins, and maresins, derived from omega-3 unsaturated fatty acids $(2,4)$, as well as other pro-resolution mechanisms (5). Resolvins not only regulate the resolution of acute inflammation but also inhibit inflammatory pain through direct actions on nociceptors through specific receptors; activation of ChemR23 by resolvin E1 potently inhibits transient receptor potential ion channel V1 (TRPV1), a key ion channel for pain transduction (4).

In contrast to acute inflammation, chronic inflammation is often detrimental, leading to a host of diseases such as periodontitis, atherosclerosis, rheumatoid arthritis, and even cancer (2). It is unclear whether chronic inflammation is also as critical for driving chronic pain as acute inflammation is for acute pain. Pain research in the past several decades has established that

\footnotetext{
${ }^{1}$ Department of Anesthesiology, Duke University Medical Center, Durham, NC 27710, USA. '2Department of Neurobiology, Duke University Medical Center, Durham, NC 27710, USA. ${ }^{3}$ Institute of Neurobiology, Institutes of Brain Science and State Key Laboratory of Medical Neurobiology, Collaborative Innovation Center for Brain Science, Fudan University, Shanghai 200032, China.

*Corresponding author: Email: ru-rong.ji@duke.edu
}

neuronal plasticity is a key mechanism for the development and maintenance of chronic pain $(1,6)$. Peripheral sensitization in nociceptors is essential for the development of chronic pain (3) and transition from acute pain to chronic
Injury/insult

Arthritis, nerve injury, cancer treatment
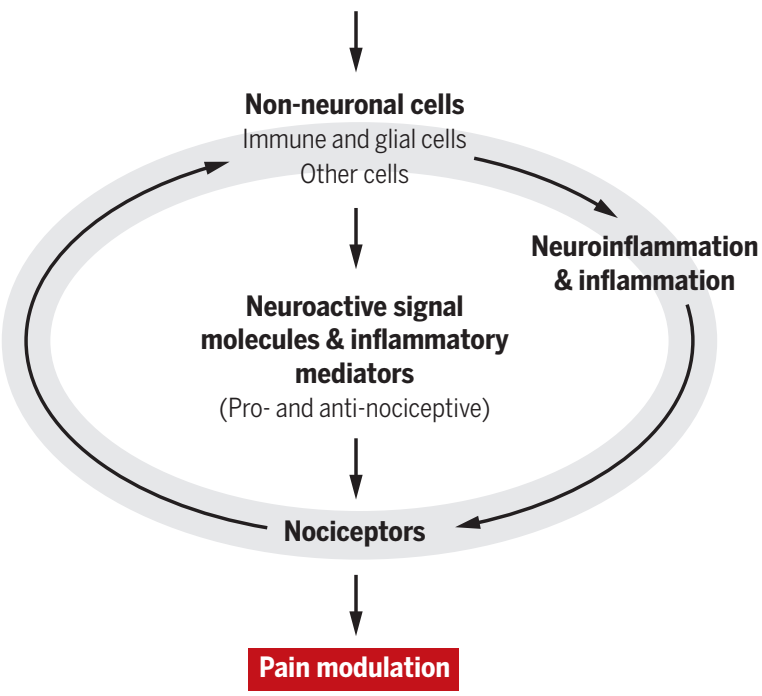

Fig. 1. Interactions between non-neuronal cells, neurons, and inflammation/neuroinflammation in different pain conditions after injury and insult. Non-neuronal cells can modulate pain in different directions by producing either pro- or anti-nociceptive mediators.

pain (7). Central sensitization (enhanced responses of pain circuits in the spinal cord and brain) regulates the chronicity of pain, causes the spread of pain beyond the site of injury, and influences the emotional and affective aspects of pain (8).

Neuroinflammation is a localized inflammation occurring in the PNS and central nervous system (CNS) in response to trauma, neurodegeneration, bacterial or viral infection, autoimmunity, and toxins $(2,9)$. The hallmarks of neuroinflam- mation are activation and infiltration of leukocytes, activation of glial cells, and increased production of inflammatory mediators. Neuroinflammation is also associated with changes of vascular cells that facilitate leukocyte infiltration $(2,9)$. Compared with inflammation, neuroinflammation is more persistent in chronic pain conditions and therefore plays a more important role in chronic pain maintenance (2). For example, fibromyalgia, a widespread chronic pain syndrome, is associated with small fiber neuropathy and neuroinflammation, although its correlation with systemic inflammation is unclear (10).

The interactions between inflammation and pain are bidirectional (Fig. 1). Nociceptive sensory neurons not only respond to immune signals but also directly modulate inflammation. For example, nociceptors express receptors for and respond to cytokines and chemokines and also produce these inflammatory mediators $(11,12)$. In a process called neurogenic inflammation, noxious stimulation causes nociceptors to release neuropeptides such as Substance P (SP) and calcitonin gene-related peptide (CGRP), leading to the extravasation of fluid and cells from the blood. Consistently, silencing nociceptors reduces allergic airway inflammation (13). Nociceptors also serve to dampen and constrain the immune response: Ablation of nociceptors abrogated pain during bacterial infection but concurrently worsened inflammation via CGRP (14). Activation of pain circuits also regulates neuroinflammation in the CNS, referred to as neurogenic neuroinflammation in chronic pain and neurodegenerative diseases (9).

Numerous non-neuronal cell types influence pain sensation, including immune, glial, epithelial, mesenchymal, cancer, and bacterial cells. In this Review, we focus on non-neuronal cells that interact with nociceptors in distinct anatomical compartments in the PNS and CNS (glial cells) under normal and pathological conditions (Fig. 2). Despite the diversity of these cells, the ways in which they modulate pain are surprisingly consistent. In response to an injury or insult, non-neuronal cells release neuromodulatory substances in close proximity to nociceptors, which either promote or dampen pain depending on the specific identities of the mediators involved (Figs. 1 and 2). Although the details will differ in each case, this general model will serve as a useful lens through which to examine specific non-neuronal cell types in pain.

\section{Pain modulation by non-neuronal cells Monocytes and macrophages}

Monocytes and macrophages serve three main functions in the immune system: phagocytosis, 
antigen presentation, and cytokine production. Monocytes and macrophages in the periphery play an active role in pain, exhibiting diverse mechanisms that are shaped by the causes and context of pain. In most cases, these cells produce pain through the release of proinflammatory mediators such as tumor necrosis factor (TNF) and interleukin-1 $\beta$ (IL-1 $\beta$ ) (11), resulting in enhanced pain transduction and conduction via modulation of ion channels such as transient receptor potential ankyrin 1 (TRPA1), TRPV1, and Nav1.7-1.9 (1, 2). Cell-specific depletion of proliferating monocytes and macrophages impairs the development of mechanical and thermal hypersensitivity caused by sterile incision and pathogens, in parallel with a decrement in IL-1 $\beta$ and other pro-algesic mediators at the site of inflammation (15). However, in a nerve injury model, deletion of peripheral monocytes does not affect neuropathic pain development (16). In a model of chemotherapy-induced neuropathic pain, $\mathrm{CX}_{3} \mathrm{CRI}^{+}$monocytes migrate into peripheral nerves and produce reactive oxygen species, which then elicit pain via the activation of TRPAl (17). After peripheral nerve injury, infiltration of monocytes and macrophages to the spinal cord is limited, arguing against a central role of these cells in neuro- pathic pain $(16,18)$. Conversely, monocytes and macrophages can also effect analgesia by releasing anti-inflammatory mediators such as IL-10 and SPMs, together promoting the resolution of the initial insult $(2,4)$. In support of this view, depletion of monocytes and macrophages delayed the resolution of inflammatory pain (19). Macrophages have different phenotypes related to their functional states, including proinflammatory M1-like and antiinflammatory M2-like phenotypes, which may play distinct roles in the induction and resolution of pain.

\section{T lymphocytes}

$\mathrm{T}$ cells are critical elements of adaptive immunity that have also been implicated in pain, with the most evidence for a role in neuropathic pain. After nerve injury, T cells infiltrate the dorsal root ganglia (DRG) and release the proalgesic mediator leukocyte elastase (LE), resulting in mechanical allodynia. Consistently, inhibitor of LE reduces nerve injury-induced allodynia (20). In the spinal cord, T cell infiltration occurs after nerve injury and is required for the development of mechanical hypersensitivity, as indicated by a reduction of pain behaviors in T cell-deficient Rag1-null mice (21).
A recent report supported the role of spinal $\mathrm{T}$ cells in nerve injury-induced neuropathic pain but limited this function only to female mice, with male mice instead depending on microglial signaling for pain. This intriguing finding was attributed to the sexually dimorphic expression of peroxisome proliferatoractivated receptors (PPARs) $\alpha$ and $\gamma$ in T cells (22). Different types of $\mathrm{T}$ cells play different roles in chronic pain. For example, adoptive transfer of toxic $\mathrm{T}$ cells $\left(\mathrm{CD} 8^{+} \mathrm{T}\right.$ cells) via intrathecal injection enhances neuropathic pain, whereas injection of regulatory $\mathrm{T}$ cells $\left(\mathrm{T}_{\mathrm{reg}}\right.$ cells) decreases neuropathic pain after chemotherapy (23).

\section{Keratinocytes}

Keratinocytes are the primary cells of the epidermis. They reside near the peripheral terminals of nociceptors and produce various neuroactive mediators such as adenosine $5^{\prime}$-triphosphate (ATP), IL-1 $\beta$, prostaglandin E2, endothelin, and nerve growth factor (NGF) that are known to elicit pain, suggesting that these cells can directly activate nociceptors. Photostimulation of channelrhodopsin-expressing keratinocytes is sufficient to generate nocifensive behaviors and evoke action potentials in specific subsets

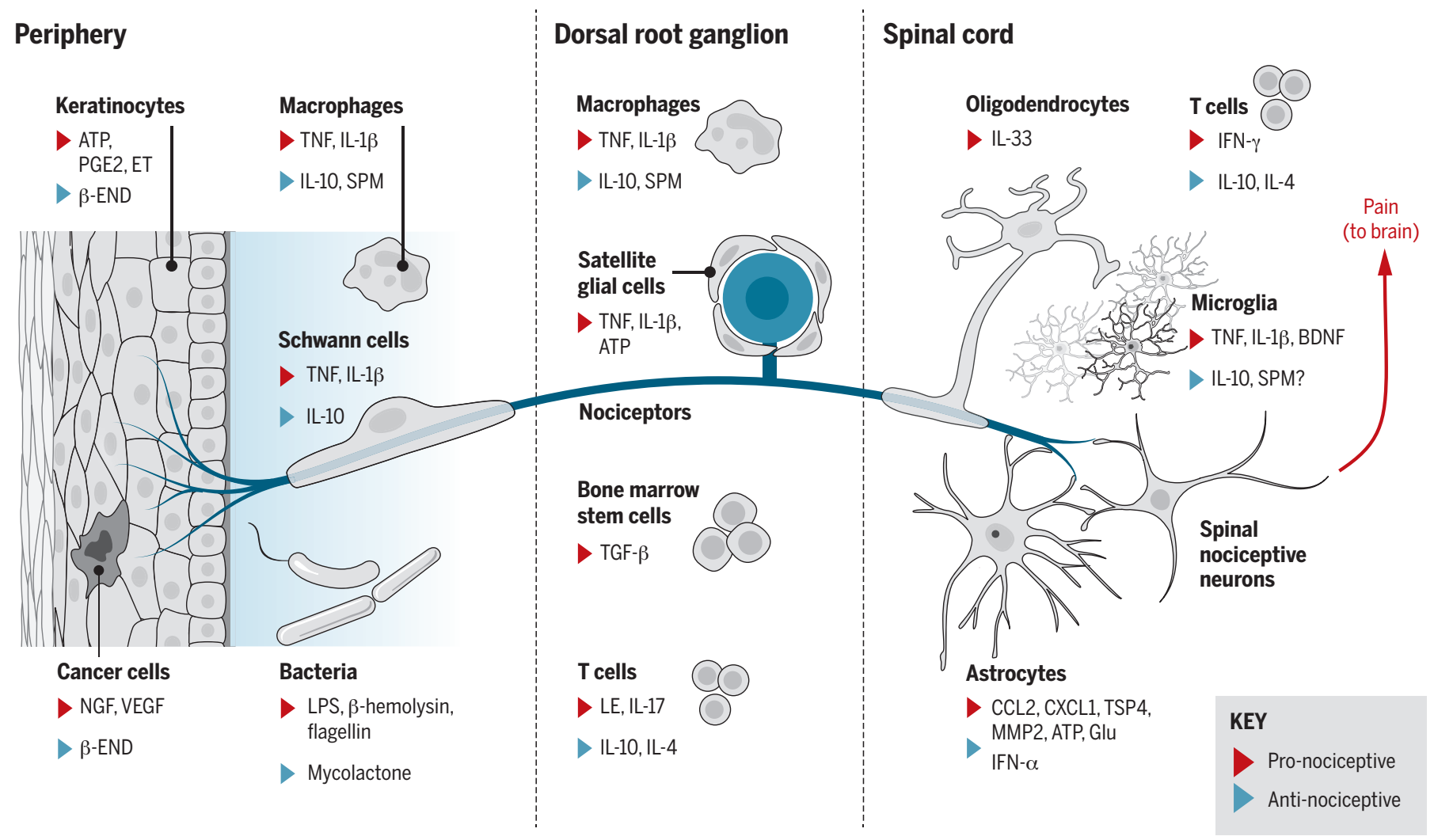

Fig. 2. Interactions between distinct parts of a nociceptor with different types of non-neuron cells. Included are keratinocytes, Schwann cells, satellite glial cells, oligodendrocytes, and astrocytes, as well as immune cells (for example, macrophages and T cells), microglia, cancer cells, and stem cells. These non-neuronal cells produce both pro-nociceptive (highlighted in red) and anti-nociceptive (highlighted in blue) mediators, which can bind their respective receptors on the nociceptor to modulate its sensitivity and excitability. The central terminal of the nociceptor forms a nociceptive synapse with a postsynaptic neuron in the spinal cord dorsal horn to mediate pain transmission in the CNS. END, endorphin; ET, endothelin; Glu, glutamate; IFN- $\alpha$, interferon- $\alpha$; LE, leukocyte elastase. 
of cutaneous nociceptive afferents (24). Keratinocytes are multifunctional, promoting pain or analgesia depending on the condition and context. The familiar experience of sunburn illustrates the dual nature of these cells. Before the pain of sunburn sets in, the feeling of sun on the skin is a pleasurable experience. Here, keratinocytes participate by releasing the endogenous opioid peptide $\beta$-endorphin ( $\beta$-END), which produces both analgesia and reward (25). After light overexposure, when inflammation and injury occur, keratinocytes also elicit pain via the activation of TRPV4 and the subsequent release of endothelin onto cutaneous nociceptors (26). Although keratinocytes are not immune cells, they can release inflammatory mediators including cytokines (27), illustrating how nonimmune cells can also regulate inflammation.
ATP, colony-stimulating factor-1 (CSF1), chemokines (CCL2 and CX3CL1), and proteases, which can originate from injured or activated sensory neurons (Fig. 3). In parallel, expression of the receptors for ATP and CX3CL1 (P2X4, P2X7, $\mathrm{P} 2 \mathrm{Y} 12$, and CX3CR1) is increased selectively on spinal microglia in response to nerve injury $(28,29)$. Activation of these receptors typically converges on an intracellular signaling cascade involving the phosphorylation of p38 mitogenactivated protein (MAP) kinase, which leads to the increased production and release of TNF- $\alpha$, IL-1 $\beta$, IL-18, and brain-derived growth factor (BDNF), as well as increased expression of cyclooxygenase (COX) and subsequent synthesis of prostaglandin $\mathrm{E}_{2}(6)$. These neuromodulators can then fine-tune both excitatory and inhibitory synaptic transmission, which ultimately enhances pain signal transmission to the brain.

lease from microglia and drives robust acute inflammatory pain behaviors before the morphological activation of microglia (36). Spinal microglia signaling is sex-dependent, and microglial signaling inhibitors such as minocycline and p38 inhibitor reduce neuropathic pain primarily in male mice, with little to no effect on female mice (22). Although microglia are critical to the development of chronic pain, they may function in the maintenance of chronic pain as well. For example, examination of microglial enhancers by using chromatin immunoprecipitation sequencing (ChIP-seq) revealed persistent alterations in close proximity to transcriptionally regulated genes, leading to the hypothesis that these changes may contribute to the "memory" recorded at a molecular level (18). Microglia are also involved in opioid-induced hyperalgesia through microglia-mediated disruption of neuronal chloride homeostasis (34). Morphine paradoxically prolongs neuropathic pain in rats, and selective inhibition of spinal cord microglia in vivo through DREADD (Designer Receptor Exclusively Activated by Designer Drugs) reverses morphine-induced pain sensitization for weeks (37). In the future, it will also be important to determine whether microglia are involved in the resolution of chronic pain, which would be expected on the basis of the dual role of their peripheral macrophage counterparts (19).

Astrocytes perform numerous critical functions such as neurotransmitter recycling, formation of the blood-brain barrier, regulation of extracellular ion concentration, and modulation of synaptic transmission, among many others. Nerve injury induces myriad changes in astrocytes that lead to enhanced pain. For example, after nerve injury, astrocytes lose their ability to maintain the homeostatic concentrations of extracellular potassium $\left(\mathrm{K}^{+}\right)$and glutamate, leading to neuronal hyperexcitability (28). Astrocytes can also signal directly

Fig. 3. Neuron-glial interactions in the spinal cord for the amplification of chronic pain. Painful injuries such as nerve injury, arthritis, cancer, and treatment (chemotherapy) cause hyperactivity of nociceptors and secretion of glial modulators from their central terminals, leading to the activation of microglia and astrocytes in the spinal cord dorsal horn. Upon activation, microglia and astrocytes secrete neuromodulators to drive chronic pain by inducing synaptic and neuronal plasticity. Pre- and postsynaptic neurons can both "listen" and "talk" to microglia and astrocytes. CASP6, caspase-6.

\section{Central glial cells: Microglia, astrocytes, and oligodendrocytes}

Microglia serve as the resident macrophages of the spinal cord and brain, and abundant evidence supports their role in pathological pain $(28,29)$. A hallmark of microglia is their rapid activation in response to even minor pathological changes in the CNS. Peripheral nerve injury induces a marked proliferation and activation of microglia in parallel with up-regulation of microglial markers such as IBA1 and CD11b in the spinal cord. Indeed, spinal microglia have been strongly implicated in the pathogenesis of neuropathic pain after nerve injury (18, 29-32). The signals that activate these microglia include
For example, TNF and IL-1 $\beta$ enhance excitatory synaptic transmission and suppress inhibitory synaptic transmission in spinal cord lamina II neurons (33). BDNF disrupts chloride homeostasis and suppresses $\gamma$-aminobutyric acid (GABA)-mediated inhibitory synaptic transmission (disinhibition) in spinal lamina I projection neurons $(6,34)$. Selective activation of spinal microglia by fractalkine (CX3CL1) is sufficient to rapidly facilitate synaptic strength between primary afferent C-fibers and lamina I projection neurons (35). Caspase-6 is only expressed in axonal terminals in the spinal cord and mediates neuron-microglial interaction. Recombinant caspase- 6 triggers marked TNF reto neurons through physically coupled networks mediated by gap junctions to facilitate intercellular transmission. Gap junction communication is mediated by connexin- 43 (Cx43), the predominant connexin expressed in astrocytes. Nerve injury induces persistent up-regulation of $\mathrm{Cx} 43$ in astrocytes and switches the function of $\mathrm{Cx} 43$ from gap junction communication to paracrine modulation (38). This paracrine regulation leads to the increased release of glutamate, ATP, and chemokines through a paracrine mechanism. The astrocyte-derived chemokines act as neuromodulators and can potentiate excitatory synaptic transmission in the spinal cord pain circuitry (38). Furthermore, nerve injury 
up-regulates CXCL13 in spinal cord neurons, which can activate astrocytes via CCR5 to maintain neuropathic pain (39). Thus, chemokines facilitate neuropathic pain via bidirectional neuron-astrocyte interactions (Fig. 3). Nerve injury also induces spinal cord and cortical astrocytes to up-regulate thrombospondin-4 (TSP4), which promotes neuropathic pain through the formation of new synapses and rewiring of somatosensory cortical circuits $(40,41)$. A single human astrocyte may contact more than 1 million synapses, and such complexity points to a more important role of astrocytes in humans that bears further investigation. As compared with microglial activation, astrocyte activation in chronic pain conditions is more persistent, indicating their contribution to the chronicity of pain (28).

Oligodendrocytes create the myelin sheath that provides support and insulation to axons in the CNS (Fig. 2). In spite of their ubiquity and importance, only recently has a role for oligodendrocytes been revealed in pain. In the chronic constriction injury model of nerve injury-induced neuropathic pain, oligodendrocyte-derived IL-33 contributes to pain hypersensitivity via MAP kinases and nuclear factor $\kappa \mathrm{B}(\mathrm{NF}-\kappa \mathrm{B})(42)$. Similarly, in post mortem spinal cord samples from HIV patients, expression of oligodendrocyte markers such as NG2, Oligo2, and platelet-derived growth factor receptor $\alpha$ (PDGFR $\alpha)$ increases, reflecting a persistent activation of oligodendrocytes in chronic pain (43). Conversely, toxinmediated ablation of oligodendrocytes induces neuropathic pain symptoms, suggesting a potential protective role of these cells (44). These divergent findings suggest that as with other non-neuronal cells, oligodendrocytes play active and context-specific roles in pain.

\section{Peripheral glia: Schwann cells and satellite glial cells}

Like their central counterparts, the major glial cells of the PNS, the Schwann cells and the satellite glial cells (SGCs), contribute to pain. In response to painful stimuli, these peripheral glial cells are activated before central glia and release various inflammatory mediators, sensitizing nociceptors at axons (Schwann cells) and cell bodies (SGCs). After nerve injury, activated Schwann cells mediate the breakdown of the blood-nerve barrier via the secretion of matrix metalloproteinase 9 (MMP-9), which promotes the recruitment of immune cells from the vasculature and their subsequent release of more pro-nociceptive mediators (27). SGCs surround the somata of DRG neurons and are directly coupled to each other via gap junctions. After nerve injury, SGCs become activated and proliferate (27). SGCs contribute to chronic pain sensitization by producing cytokines and MMPs that regulate the cleavage and activation of cytokines (28). Nociceptive activity also causes ATP release from neuronal soma to activate $\mathrm{P} 2 \mathrm{X} 7$ in SGCs, leading to TNF release from SGCs and subsequent increase in neuronal excitability (45). Activation of P2X7 receptors in SGCs also reduces pain through down-regulation of $\mathrm{P} 2 \mathrm{X} 3$ receptors in nociceptive neurons (46).

\section{Stem cells}

Bone marrow stromal cells or bone marrow stem cells (BMSCs) produce many beneficial effects to promote tissue regeneration and tissue repair by secreting growth factors. These cells can also effectively control inflammation and neuroinflammation by secreting antiinflammatory mediators. The duration of pain relief by BMSCs is remarkable after systemic or local injection $(47,48)$. A single intrathecal injection of BMSCs inhibits nerve injury-induced neuropathic pain for many weeks via secretion of transforming growth factor- $\beta 1$ (TGF- $\beta 1$ ), a potent anti-inflammatory cytokine (48). The analgesic effects of intrathecal BMSCs were abolished by a neutralizing antibody against

\section{“...non-neuronal cells can communicate with nociceptive neurons by 'listening' and 'talking' to neurons..."}

TGF- $\beta 1$. Intrathecal BMSCs effectively suppress nerve injury-induced glial activation and neuroinflammation in DRGs and spinal cord. After intrathecal injection, BMSCs migrate to DRG via a chemotaxic signal (CXCL12) triggered by nerve injury (48). This paracrine modulation of pain by BMSCs is very different from other stem cell strategies for chronic pain management. For example, implantation of forebrain GABAergic precursor cells into the spinal cord reduced nerve injury-induced neuropathic pain because these precursor cells can differentiate into functional GABAergic neurons in the spinal cord (49).

\section{Cancer cells}

Cancer pain is a complex pain state, involving inflammatory, neuropathic, compressive, and ischemic mechanisms. Cancer pain is commonly caused by tumors that metastasize from distant sites to the bone and by tumors infiltrating the nerve. Cancers generate and secrete algogenic mediators (such as protons, bradykinin, endothelins, prostaglandins, and proteases) that sensitize and activate nociceptors in the cancer microenvironment (50). In particular, cancer cells secrete NGF and vascular endothelial growth factor (VEGF), which induce hyperinnervation of pain-mediating nerve fibers in cancer tissues $(50,51)$. VEGF promotes cancer pain through a nonvascular regulation. VEGF receptor 1 (VEGFR1) is expressed in nociceptors, and tumor-derived VEGF family members increase nociceptor excitability and produce pain hypersensitivity through selective activation of VEGFR1 (51). Certain cancer cells, such as Walker-256 rat mammary gland carcinoma cells, also secrete TGF- $\beta$. In early stages of cancer, TGF- $\beta$ acts as a tumor suppressor through antiproliferative and proapoptotic actions. During tumor progression, TGF- $\beta$ becomes an oncogenic factor and causes angiogenesis, immunosuppression, and proliferation, invasion, and metastasis of cancers. TGF- $\beta 1$ also promotes bone cancer pain (52). Thus, this anti-inflammatory cytokine might have different roles in different pain conditions via different signaling mechanisms. Microglia also play an active and distinct role in bone cancer pain. Spinal microglia contribute to the maintenance of bone cancer pain in female rats (53). Bone cancer causes increased ATP secretion in cerebrospinal fluid and upregulation of $\mathrm{P} 2 \mathrm{X} 7$ receptor in spinal microglia. Activation of P2X7 results in increased synthesis and release of IL-18 via p38 MAP kinase. Spinal inhibition of the P2X7/p-38/IL-18 pathway reduces the advanced phase of bone cancer pain and suppresses hyperactivity of spinal-wide dynamic-range neurons (53). This result suggests that spinal cord microglia have an active role in late-phase bone cancer pain in female rats. Further investigation is warranted to test sex-dependent regulation of microglial signaling in different animal models and also in different phases of chronic pain. Some cancers such as melanoma are not painful in the majority of patients. It is possible that melanoma may produce some analgesic molecules such as $\beta$-endorphin to suppress pain (Fig. 2), which can promote the optimal growth of cancers, dampening the warning function of pain.

\section{Pain modulation by bacterial infections through direct interactions with sensory neurons}

Infections caused by bacterial, fungal, and viral pathogens are commonly associated with pain. Until recently, it was generally believed that infections cause pain in an indirect manner through the intermediating effects of immune cells and the inflammatory substances they secrete (Fig. 4). However, emerging evidence suggests that pathogens can also directly activate nociceptors and elicit pain using a suite of previously unknown and intriguing mechanisms (14). For example, the bacterium Staphylococcus aureus possesses a pore-forming toxin called $\alpha$-hemolysin, which enables it to destroy host cells and also to cause pain by directly forming pores in nociceptors, permitting cation influx and action potential firing through metallopeptidase A disintegrin and metalloproteinase domain-containing protein 10 (ADAM10) (14). Lipopolysaccharide (LPS) is the best-studied element derived from bacterial cell walls and is also known to produce pain hypersensitivity by sensitizing TRPV1 in nociceptors (54). Bacteria also produce pain by secreting substances that are ligands for receptors on nociceptors, as is the case with N-formyl peptides, which are metabolic by-products of bacteria that activate nociceptors and elicit pain by binding to the formyl peptide receptor 1 (FPR1), a $\mathrm{G}$ protein-coupled receptor. The pain produced 
through these mechanisms is independent of the immune response (14). Bacterial infection is not always painful, however. Mycobacterium ulcerans, the etiological agent of Buruli ulcer, causes extensive skin lesions but not pain. $M$. ulcerans produces remarkable analgesia, through a specific activation of sensory neurons triggered by the secreted mycobacterial polyketide mycolactone. Mycolactone elicits analgesia through activation of type 2 angiotensin II receptors (AT2Rs), leading to the activation of K2P potassium channels TRAKK (KCNK4) and hyperpolarization of nociceptors (55).

Although viruses are not cells, viral infections are frequently associated with inflammation and pain, such as in sore throat. Several types of viruses-such as herpes simplex virus 1 (HSV-1), HSV-2, and VZV (varicella zoster virus) - can infect sensory neurons to produce painful syndromes. (pathogen-activated molecular patterns) but also by DAMP (danger-activated molecular patterns), the endogenous ligands released after tissue injury. Activation of TLR4 in spinal microglia and astrocytes critically contributes to the development and maintenance of inflammatory and neuropathic pain, as well as opioidinduced hyperalgesia, by eliciting the synthesis of TNF- $\alpha$ and IL- $1 \beta(37,56,57)$. Secretion of these cytokines interacts with nociceptive neurons in the spinal cord pain circuitry (Fig. 4).

Primary sensory neurons in the DRG and trigeminal ganglia also express TLRs $(54,58-60)$. TLRs are differentially expressed in primary sensory neurons and modulate different sensory functions. TLR4 appears to be functionally coupled with TRPV1 in nociceptors, so that LPS binding to TLR4 increased $\mathrm{Ca}^{2+}$ influx in sensory neurons $(54,60)$. In contrast to its intracellu- port of certain small molecules across the neuronal membrane through a to-be-determined mechanism. This A $\beta$-fiber-specific transport process was exploited to deliver the membraneimpermeable anesthetic QX-314, resulting in selective blockade of A-fibers. Silencing A-fibers in this manner revealed that these neurons inhibit pain under normal conditions but give rise to mechanical allodynia in chemotherapyinduced neuropathy (59), in support of the "Gate Control Theory." Further studies are warranted to investigate the ion channels and signaling molecules that give rise to this previously unknown function of TLR5.

Whereas coupling of TLRs with ion channels in nociceptors is important for the induction of acute pain, activation of conventional TLR signaling in nociceptors through myeloid differentiation factor 88 (MyD88), a key downstream
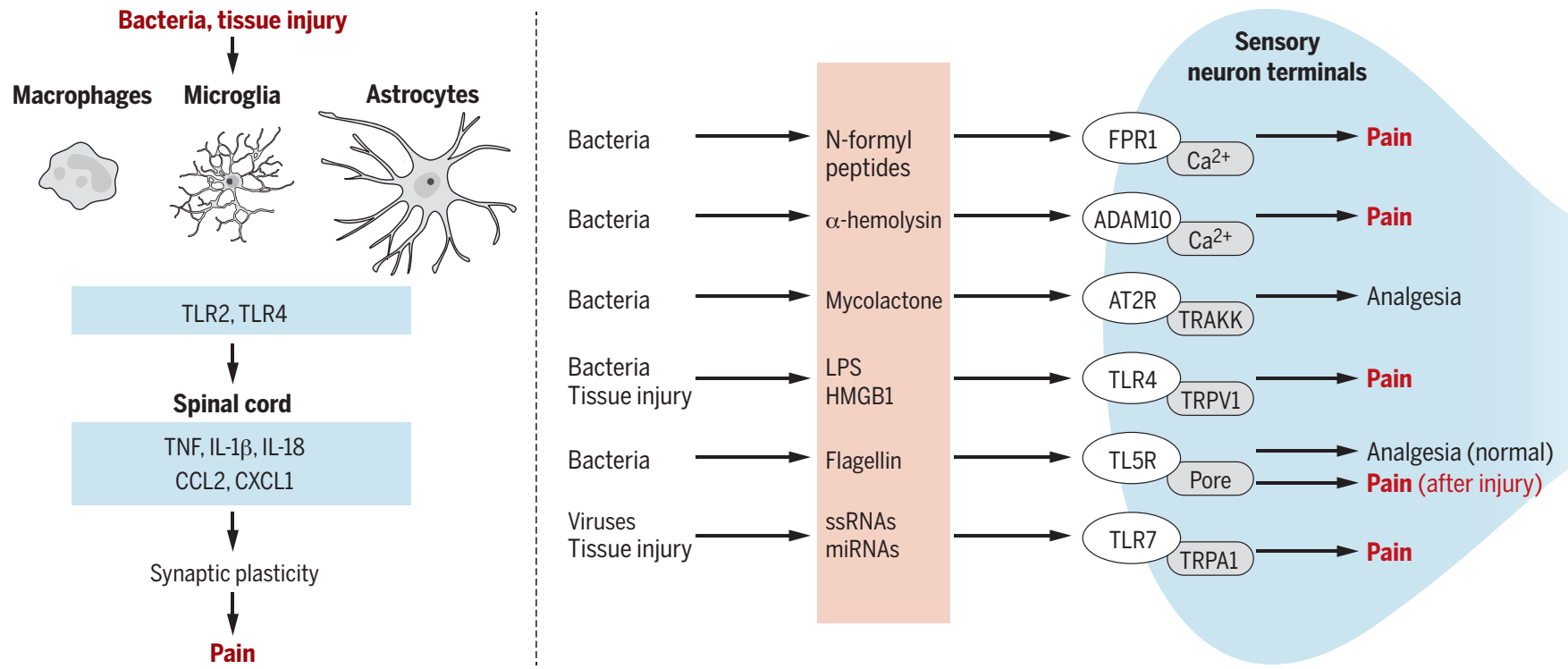

Fig. 4. Infections and tissue injury regulate pain via both neuronal and non-neuronal mechanisms through TLRs. Bacterial infections can modulate pain through direct interactions with specific receptors such as FPR and TLR on primary sensory neurons, including nociceptors and mechanoceptors, leading to increased and decreased pain sensitivity. TLRs are expressed by neurons, glial cells, and immune cells and are activated by PAMP (infections), as well as DAMP, endogenous TLR ligands (such as HMGB1 or miRNAs) released after tissue injury. HMGB1, high mobility group box 1 protein; ssRNA, single-stranded RNA.

Shingles, also called herpes zoster, is a painful skin rash and usually appears in a defined region of face or body that corresponds to dermatomes. It is most common in older adults and people who have weak immune systems. Viral infections may activate Toll-like receptors (TLRs) in nociceptors to modulate pain and neuroinflammation.

\section{Toll-like receptors mediate bilateral neuron-immune and neuron-glial interactions}

Mounting evidence suggests that TLRs are instrumental in bilateral interactions between neurons and non-neuronal cells (Fig. 4). TLRs are typically expressed by immune and glial cells to regulate innate immunity through the activation of the NF- $\mathrm{KB}$ and MAP kinase signaling pathways. TLRs are activated not only by PAMP lar localization in immune cells, TLR7 expresses on the cell surface as well as in axons of DRG nociceptors. Activation of TLR7 by synthetic ligands causes immediate activation and excitation of nociceptors (61). MicroRNA (miRNA) let-7b is an endogenous ligand of TLR7 and activates TLR7 through the GUUGUGU motif. Let-7b causes rapid activation and excitation of nociceptors through the coupling of TLR7 and TRPA1. Consistently, intraplantar injection of let-7b elicits rapid spontaneous pain through TLR7 and TRPA1 (58).

TLR5 is expressed by large-sized A-fibers, especially $A \beta$ fibers that normally mediate touch sensation. The canonical ligand for TLR5 is flagellin, a globular protein that forms the filament in bacterial flagella. In large A-fibers, activation of TLR5 by flagellin permits the rapid trans- signaling molecule for TLRs, is required for the development of chronic pain after painful insults $(23,58)$. Deletion of MyD88 in nociceptors results in deficits in neuropathic pain as well as in innate and adaptive immunity in the PNS after chemotherapy-induced neuropathy (23). Thus, nociceptors share similar features with immune cells by expressing TLRs, cytokines, chemokines, and inflammatory cascades (62). Through the coupling with ion channels, nociceptor TLRs act as a distinct class of "danger receptors" for the rapid detection of PAMP and DAMP. Thus, primary sensory neurons not only sense pain, itch, temperature, and touch (63) but also sense "danger" (DAMP and PAMP, which are partially overlapped with algogenic and prurigogenic agents), and this danger sensation will trigger protective responses such as a rapid 
withdrawal or scratching response (61) as well as delayed innate and adaptive immunity responses through MyD88 signaling in sensory neurons (23). This evolutionarily conserved signaling pathway persists because of its critical biological function, but excessive activity of the same pathway can also lead to autoimmune diseases if left unchecked.

\section{Concluding remarks and future directions}

Chronic pain is a rising health concern worldwide, affecting up to $30 \%$ of adults. Despite this enormous burden, the current treatments for chronic pain are inadequate. Chronic pain typically includes inflammatory pain after tissue injury, cancer pain, and neuropathic pain after nerve injury as a result of diabetic neuropathy, viral infection, or major surgeries. Common treatments, such as chemotherapy and chronic opioid treatment, also cause chronic pain. In the past decade, great progress has been made to demonstrate the critical roles of glial cells and immune cells-as well as keratinocytes, cancer cells, and stem cells-in the pathogenesis and resolution of chronic pain. These non-neuronal cells can communicate with nociceptive neurons by "listening" and "talking" to neurons, modifying their activities by secreting neuroactive signaling molecules that can be both pro- and anti-nociceptive (Figs. 1 to 3). There is increasing appreciation that many inflammatory mediators such as cytokines and chemokines are indeed neuromodulators in the PNS and CNS (2). Thus, chronic pain could be caused by both "neuropathy" and "gliopathy" (28), as well as dysregulations of other non-neuronal cells (Fig. 2). We extend the boundary of non-neuronal cells to include immune cells, cancer cells, and stem cells because they can infiltrate damaged or normal tissues to interact with nociceptors and also produce neuroactive modulators to alter pain states. Given the close link between inflammation and pain, we discussed non-neuronal cells in the context of inflammation and especially neuroinflammation (2) that is tightly associated with chronic pain. Inflammation and neuroinflammation can be modulated not only by immune cells but also by other non-neuronal cells such as keratinocytes, stem cells, and cancer cells and even by nociceptive neurons in the PNS and CNS. Infections by bacteria and other pathogens not only affect immune and glial cells but also have a direct impact on sensory neurons, eliciting rapid nociceptive responses through activation of neuronal receptors such as FRP1 and TLRs. In particular, TLRs in immune or glial cells and sensory neurons not only sense the danger signals from tissue injury and infections but also regulate bilateral interactions between neurons and neuron-neuronal cells, and these interactions are important for the induction and chronicity of inflammation and pain.

Although several types of non-neuronal cells contribute to the pathogenesis of pain, deletion of a cell type entirely could be detrimental. We should bear in mind that these non-neuronal cells, except for cancer cells, are protective in the normal conditions but dysregulated in pathological conditions. Although blocking their function may help pain relief for a short period, restoring their normal function through pro-resolution approaches is probably the best way. On the basis of this principle, we propose the following therapeutic strategies as future directions. First, different pharmaceutical strategies should be considered at different development stages of pain and inflammation. Anti-inflammatory drugs such as inhibitors of TNF are more effective in the early stage of pain and inflammation. Resolution of inflammation is a new therapeutic frontier (5), and SPMs such as resolvins and protectins may promote the resolution of inflammation and pain, which can be partially achieved via dietary control (2). In particular, neuroprotectin D1 is effective in controlling neuroinflammation and neuropathic pain (64). Second, cell therapies such as implantation of BMSCs are promising, demonstrating long-term pain relief and even final resolution of pain $(47,48)$. However, only a very small percentage of cells in bone marrow are stem cells for autologous transplantation. Instead, macrophages are an abundant cell source in blood, and implantation of resident and M2-like macrophages could be an alternative to promote the resolution of inflammation and pain. Alternatively, inducible pluripotent stem cells (iPSCs) from abundant cells (such as skin or blood) could be transformed into the desired cell type and then transplanted. Moreover, transcription activator-like effector nuclease (TALEN)- or clustered regularly interspaced short palindromic repeats (CRISPR)-mediated gene editing ex vivo could first be used to enhance the production of pro-resolution mediators and the migration capability of the cells to be transplanted so that they can target the damaged tissue more accurately. Last, neuromodulation such as vagus nerve stimulation and acupuncture has been used for inflammation control and pain treatment $(65,66)$. In addition to local acute analgesia via adenosine from non-neuronal cells (67), acupuncture and sciatic nerve activation also regulate inflammation through vagus nerve activation and dopamine release (68). It will be of great interest to test whether neuromodulation can also help to restore the normal function of non-neuronal cells in chronic pain as a nonpharmaceutical approach.

\section{REFERENCES AND NOTES}

1. A. I. Basbaum, D. M. Bautista, G. Scherrer, D. Julius, Cell 139, 267-284 (2009)

2. R. R. Ji, Z. Z. Xu, Y. J. Gao, Nat. Rev. Drug Discov. 13, 533-548 (2014).

3. M. S. Gold, G. F. Gebhart, Nat. Med. 16, 1248-1257 (2010)

4. Z. Z. Xu et al., Nat. Med. 16, 592-597, 1p, 597 (2010).

5. J. N. Fullerton, D. W. Gilroy, Nat. Rev. Drug Discov. 15, 551-567 (2016).

6. J. A. Coull et al., Nature 438, 1017-1021 (2005).

7. D. B. Reichling, J. D. Levine, Trends Neurosci. 32, 611-618 (2009).

8. C. J. Woolf, M. W. Salter, Science 288, 1765-1768 (2000).

9. D. N. Xanthos, J. Sandkühler, Nat. Rev. Neurosci. 15, 43-53 (2014).

10. N. Üçeyler et al., Brain 136, 1857-1867 (2013).

11. M. Zelenka, M. Schäfers, C. Sommer, Pain 116, 257-263 (2005).
12. F. A. White et al., Proc. Natl. Acad. Sci. U.S.A. 102 14092-14097 (2005)

13. S. Talbot et al., Neuron 87, 341-354 (2015).

14. I. M. Chiu et al., Nature 501, 52-57 (2013).

15. N. Ghasemlou, I. M. Chiu, J. P. Julien, C. J. Woolf, Proc. Natl. Acad. Sci. U.S.A. 112, E6808-E6817 (2015).

16. J. Peng et al., Nat. Commun. 7, 12029 (2016)

17. E. A. Old et al., J. Clin. Invest. 124, 2023-2036 (2014).

18. F. Denk, M. Crow, A. Didangelos, D. M. Lopes, S. B. McMahon, Cell Reports 15, 1771-1781 (2016).

19. H. L. Willemen et al., J. Pain 15, 496-506 (2014).

20. L. Vicuña et al., Nat. Med. 21, 518-523 (2015).

21. M. Costigan et al., J. Neurosci. 29, 14415-14422 (2009).

22. R. E. Sorge et al., Nat. Neurosci. 18, 1081-1083 (2015).

23. X. J. Liu et al., Cell Res. 24, 1374-1377 (2014).

24. K. M. Baumbauer et al., elife 4, e09674 (2015).

25. G. L. Fell, K. C. Robinson, J. Mao, C. J. Woolf, D. E. Fisher, Cell 157, 1527-1534 (2014).

26. C. Moore et al., Proc. Natl. Acad. Sci. U.S.A. 110, E3225-E3234 (2013).

27. M. Calvo, J. M. Dawes, D. L. Bennett, Lancet Neurol. 11 629-642 (2012)

28. R. R. Ji, T. Berta, M. Nedergaard, Pain 154 (suppl. 1), S10-S28 (2013)

29. P. M. Grace, M. R. Hutchinson, S. F. Maier, L. R. Watkins, Nat. Rev. Immunol. 14, 217-231 (2014).

30. M. Tsuda et al., Nature 424, 778-783 (2003).

31. A. K. Clark et al., Proc. Natl. Acad. Sci. U.S.A. 104 10655-10660 (2007).

32. Z. Guan et al., Nat. Neurosci. 19, 94-101 (2016).

33. Y. Kawasaki, L. Zhang, J. K. Cheng, R. R. Ji, J. Neurosci. 28 5189-5194 (2008)

34. F. Ferrini et al., Nat. Neurosci. 16, 183-192 (2013).

35. A. K. Clark et al., J. Neurosci. 35, 4552-4570 (2015).

36. T. Berta et al., J. Clin. Invest. 124, 1173-1186 (2014).

37. P. M. Grace et al., Proc. Natl. Acad. Sci. U.S.A. 113, E3441-E3450 (2016).

38. G. Chen et al., Brain 137, 2193-2209 (2014).

39. B. C. Jiang et al., J. Clin. Invest. 126, 745-761 (2016).

40. D. S. Kim et al., J. Neurosci. 32, 8977-8987 (2012).

41. S. K. Kim et al., J. Clin. Invest. 126, 1983-1997 (2016).

42. A. C. Zarpelon et al., FASEB J. 30, 54-65 (2016).

43. Y. Shi, J. Shu, Z. Liang, S. Yuan, S. J. Tang, Mol. Pain 12, 1 (2016)

44. S. Gritsch et al., Nat. Commun. 5, 5472 (2014).

45. X. Zhang, Y. Chen, C. Wang, L. Y. Huang, Proc. Natl. Acad. Sci. U.S.A. 104, 9864-9869 (2007).

46. Y. Chen et al., Proc. Natl. Acad. Sci. U.S.A. 105, 16773-16778 (2008).

47. W. Guo et al., Stem Cells 29, 1294-1303 (2011).

48. G. Chen, C. K. Park, R. G. Xie, R. R. Ji, J. Clin. Invest. 125 3226-3240 (2015)

49. J. M. Bráz et al., Neuron 74, 663-675 (2012).

50. P. Mantyh, Pain 154 (suppl. 1), S54-S62 (2013).

51. D. Selvaraj et al., Cancer Cell 27, 780-796 (2015).

52. Q. Xu et al., J. Neurosci. 33, 19099-19111 (2013).

53. Y. Yang et al., J. Neurosci. 35, 7950-7963 (2015).

54. A. Diogenes, C. C. Ferraz, A. N. Akopian, M. A. Henry, K. M. Hargreaves, J. Dent. Res. 90, 759-764 (2011).

55. E. Marion et al., Cell 157, 1565-1576 (2014).

56. F. Y. Tanga, N. Nutile-McMenemy, J. A. DeLeo, Proc. Natl. Acad. Sci. U.S.A. 102, 5856-5861 (2005)

57. C. A. Christianson et al., Pain 152, 2881-2891 (2011)

58. C. K. Park et al., Neuron 82, 47-54 (2014).

59. Z. Z. Xu et al., Nat. Med. 21, 1326-1331 (2015).

60. Y. Li et al., J. Neurosci. 35, 13487-13500 (2015).

61. T. Liu, Z. Z. Xu, C. K. Park, T. Berta, R. R. Ji, Nat. Neurosci. 13 1460-1462 (2010).

62. I. M. Chiu, C. A. von Hehn, C. J. Woolf, Nat. Neurosci. 15, 1063-1067 (2012)

63. A. Zimmerman, L. Bai, D. D. Ginty, Science 346, 950-954 (2014)

64. Z. Z. Xu et al., Ann. Neurol. 74, 490-495 (2013).

65. L. V. Borovikova et al., Nature 405, 458-462 (2000).

66. M. D. da Silva et al., Mol. Neurobiol. 51, 19-31 (2015).

67. N. Goldman et al., Nat. Neurosci. 13, 883-888 (2010).

68. R. Torres-Rosas et al., Nat. Med. 20, 291-295 (2014).

\section{ACKNOWLEDGMENTS}

Our research is supported by NIH (R01 grants DE17794, DE22743, and NS87988 to R.-R.J.) and National Natural Science Foundation of China (grant 31420103903 to Y.-Q.Z and R.-R.J.). All the figures have not been published before.

\subsection{6/science.aaf8924}




\section{Science MIAAAS}

EXTENDED PDF FORMAT SPONSORED BY

Sample-Size Antibodies Now Available

n) Learn More RD DYSTEMS

www.rndsystems.com
Pain regulation by non-neuronal cells and inflammation

$\mathrm{Ru}$-Rong Ji, Alexander Chamessian and Yu-Qiu Zhang (November $3,2016)$

Science 354 (6312), 572-577. [doi: 10.1126/science.aaf8924]

Editor's Summary

This copy is for your personal, non-commercial use only.

Article Tools Visit the online version of this article to access the personalization and article tools:

http://science.sciencemag.org/content/354/6312/572

Permissions Obtain information about reproducing this article:

http://www.sciencemag.org/about/permissions.dtl

Science (print ISSN 0036-8075; online ISSN 1095-9203) is published weekly, except the last week in December, by the American Association for the Advancement of Science, 1200 New York Avenue NW, Washington, DC 20005. Copyright 2016 by the American Association for the Advancement of Science; all rights reserved. The title Science is a registered trademark of AAAS. 